\title{
Longevity and mortality in Kennel Club registered dog breeds in the UK in 2014

\author{
T. W. Lewis ${ }^{1,2^{*}}$, B. M. Wiles ${ }^{1}$, A. M. Llewellyn-Zaidi ${ }^{3}$, K. M. Evans ${ }^{1,2}$ and D. G. O'Neill ${ }^{4}$
}

\begin{abstract}
Background: The domestic dog is one of the most diverse mammalian species, exhibiting wide variations in morphology, behaviour and morbidity across breeds. Therefore, it is not unexpected that breeds should also exhibit variation in mortality and longevity. While shorter longevity per se may not necessarily be a welfare issue, a generally foreshortened lifespan in a breed that is accompanied by a high prevalence of a particular cause of death may reveal potentially serious welfare concerns and highlight scope to improve breed welfare. Survey data gathered directly from owners offer useful insights into canine longevity and mortality that can support the overall evidence base for welfare reforms within breeds.

Results: Mortality data on 5663 deceased dogs registered with the UK Kennel Club were collected from an ownerbased survey. The most commonly reported causes of death were old age (13.8\%), unspecified cancer (8.7\%) and heart failure (4.9\%); with $5.1 \%$ of deaths reported as unknown cause. Overall median age at death was 10.33 years (interquartile range: 7.17-12.83 years). Breeds varied widely in median longevity overall from the West Highland Terrier (12.71 years) to the Dobermann Pinscher ( 7.67 years). There was also wide variation in the prevalence of some common causes of death among breeds, and in median longevity across the causes of death.

Conclusion: Substantial variation in the median lifespan and the prominent causes of death exists across breeds. This study has identified some breeds with both a low median lifespan and also a high proportional mortality for one or more specific causes of death that should be considered as both potential welfare concerns as well as opportunities for improvement.
\end{abstract}

Keywords: Pedigree, Lifespan, Purebred, Death, Healthspan, Predisposition, Mortality, Longevity

\section{Plain English Summary}

The domestic dog is one of the most diverse mammalian species, exhibiting wide variations in size and shape, common diseases, and behaviours across breeds. Therefore, it is not unexpected that breeds should also exhibit differences both in how long they generally live and their common causes of death. Shorter life alone may not necessarily be viewed as a particular problem; for example, a generally healthy life followed by a fairly rapid decline might be considered a better outcome for the dog than a longer life but with a long and painful decline preceding death. However, an excessively short life in a breed that commonly dies from a particular cause of

\footnotetext{
* Correspondence: Tom.Lewis@thekennelclub.org.uk

${ }^{1}$ The Kennel Club, Clarges Street, London W1J 8AB, UK

${ }^{2}$ School of Veterinary Medicine and Science, The University of Nottingham, Sutton Bonington Campus, Sutton Bonington, Loughborough LE12 5RD, UK Full list of author information is available at the end of the article
}

death may reveal potentially serious welfare concerns. Survey data gathered directly from owners are a useful means to explore longevity and mortality in dogs.

This study collected information on 5663 deaths of dogs registered with the UK Kennel Club from an owner survey. The most common causes of death were old age (13.8\%), unspecified cancer $(8.7 \%)$ and heart failure (4.9\%); with $5.1 \%$ deaths reported as unknown cause. The overall median average lifespan was 10 years and 4 months but this varied widely across breeds from the West Highland Terrier (12 years 8.5 months) to the Dobermann Pinscher (7 years 8 months). Breeds also differed in their most common causes of death, suggesting that some breeds are susceptible to particular causes of death. There was also variation in the median age at death from the most common causes of death. This information is important because diseases associated with death in younger dogs can be seen as depriving 
more years of potential life and therefore be considered as having higher welfare impacts.

This study identified substantial variation in lifespan and the prominent causes of death across breeds. Awareness of expected lifespans across breeds may help owners to prepare for the eventual loss of their dog and even assist decision-making when selecting a breed in the first place. Breeds with short lifespans that commonly die of one or more specific causes of death were identified, and highlighted as potential welfare concerns.

\section{Background}

The domestic dog (Canis familiaris) is the most phenotypically diverse mammalian species [1]. Wide variations in behaviour $[2,3]$ and morbidity [4-6] have been reported between breeds. It is therefore not unexpected that variation in longevity should also exist across breeds, and there is some prior evidence to this effect. The median longevity of breeds under first opinion veterinary care in the UK ranged from 14.2 years in the Miniature Poodle to 5.5 years in the Dogue de Bordeaux [7] while breeds under referral care in the US ranged from 9.3 years for the Miniature Poodle to 3.5 years for the Rottweiler [8]. Surveys of owners of Kennel Club registered dogs reported median longevity ranges from 15.5 in the Lakeland Terrier to 3.8 in the Dogue de Bordeaux in the UK [9] and from 12.0 in the Shetland Sheepdog to 7.0 in the Bernese Mountain Dog in Denmark [10]. Accurate, up-to-date and representative data on canine welfare-related parameters including longevity are needed to support evidence-based efforts to understand and improve breed-related health and welfare in dogs [11]. Therefore, it is timely to report a more contemporaneous evaluation of longevity in UK Kennel Club registered dogs.

In addition to longevity, accurate information on the common causes of death in dogs (i.e. mortality) can assist prioritisation of disease-specific reforms that may extend life or improve welfare by palliating the dying process or facilitating earlier diagnosis [7]. A previous survey of pedigree dog owners in the UK identified cancer $(27 \%)$, 'old age' (18\%) and cardiac conditions (11\%) as the most common causes of death [9]. The most common causes of death in dogs under first opinion veterinary care in the UK were neoplastic diseases (16.5\%), musculoskeletal disorders (11.3\%) and neurological disorders (11.2\%) [7], while the most common causes of death in kennel club registered dogs in Denmark were 'old age' (20.4\%), cancer (14.5\%) and behaviour problems (6.4\%) [10]. These studies highlight some common trends that would benefit from more recent data relating to the current UK population of pedigree dogs. Furthermore, just as a generally shorter lifespan of some breeds compared to others has been reported [7,9], there is also some evidence from single-breed studies that proportional mortality from various causes of death varies across breeds [12-14] and this would benefit from exploration in a multi-breed study to compare the common causes of death across breeds.

Much of the available evidence on longevity and mortality of dogs is based on veterinary or insurance data that was not originally recorded for research purposes [7]. Primary-care practice data closely represent the general dog population, including a broad range of causes of death and contemporaneous recording at the point of death by the veterinarian to reduce the effects of recall bias [15]. However, limited clinical work-up may lead to some disease misclassification and there are also substantial technical complexities related to the acquisition, management and analysis of large primary-care practice datasets [15]. Pet insurance data have also been used for longevity and mortality research [16-18] but insurance data are limited by biases from owner demographics, older animals often becoming uninsured, excluded conditions, threshold financial excesses for claims and age-limited life cover $[19,20]$. Veterinary clinical data and insurance data cover both Kennel Club registered (i.e. pedigree) and non-Kennel Club registered purebred dogs that cannot generally be distinguished and analysed separately within these datasets. Therefore, inference for owners of pedigree dogs from results based on these data resources may be confounded by beliefs about differing health status between the registered and non-registered subsets of purebred dogs. Data collected specifically on Kennel Club registered dogs would allow results that pertain directly to the pedigreed segment of the overall dog population.

Longevity reflects the period between the date of birth and the date of death [7]. Data on the date of birth is routinely recorded by breed and kennel club registries close to the time of birth and also by veterinary practices and insurance companies at variable time points in a dog's life [7, 10, 17]. Unfortunately, the date and cause of death in dogs is less commonly reported to, or recorded by, breed registries, insurance companies or often even veterinary practices and therefore it has historically been challenging to access longevity and mortality data for large and representative cohorts of dogs from secondary data sources [7, 20]. An alternative research option is to institute a primary data collection process that collects information on the cause of, and age at, death of dogs directly from owners. This approach was successfully used in 2004 when the Kennel Club/British Small Animal Veterinary Association (BSAVA) Committee with the Epidemiology Unit at the Animal Health Trust carried out a nationwide direct survey of UK pedigree dog owners to identify the most common causes of death in UK dog breeds [9]. This approach offers relatively 
large volumes of survival data on breeds that are known to be pedigree but also accepts the well-recognised shortcomings to owner surveys, including variable response rates, recall and selection biases and difficulties in validation that all require strong study planning to limit or understand their impacts [15]. In the end, results from well-designed owner surveys can add unique insights that can complement other data sources to build more accurate overall representations [15].

This paper reports the results of longevity and mortality analyses from a large survey of owners of Kennel Club registered dogs in the UK carried out in 2014. The objectives of the study were to report the longevity and the most common causes of death both overall and within-breed from Kennel Club registered pedigree dogs that died over the preceding 10 years. The study aimed to add to the extant body of evidence on canine longevity and mortality and provide a reliable evidence base to support welfare reforms within breeds.

\section{Methods}

\section{Survey}

A mortality survey on previously-owned Kennel Club registered dogs was undertaken in conjunction with a larger survey of owners of living pedigree dogs registered with the Kennel Club. The methods and results of this larger survey have previously been reported [4]. In brief, the surveys were applied online using a web-based survey tool (SurveyMonkey) and were open from 8th November 2014 until 31st December 2014. There were 546,836 invitations emailed to owners of Kennel Club registered dogs. The surveys were also promoted on social media (Twitter and Facebook), the Kennel Club website, in the dog press (Dog World and Our Dogs) and within breeds by personal communication from Breed Health Coordinators. Participants in the mortality survey were asked to provide details on Kennel Club registered dogs they had owned and which had died during the 10-year period prior to the survey (1st January 2005 to 25th December 2014) with no stipulations made on date of birth. Dog owners were able to participate in either the mortality survey or the morbidity survey, or both, but dogs for which participants provided mortality information were necessarily different to those still living for which they may have provided morbidity information. The section of the questionnaire relevant to mortality contained nine additional questions, including the identity of deceased $\operatorname{dog}(\mathrm{s}$ ) (Kennel Club name and number), date of death, cause(s) of death (recordable in free-text format), whether death was unassisted or euthanasia, and whether a post mortem was performed.

\section{Data processing}

The online survey closed on 31st December 2014 and the data were exported from SurveyMonkey to a spreadsheet in Microsoft Excel CSV format for cleaning and verification against the Kennel Club database in Microsoft Access (Microsoft Corporation, 2017). Each dog was linked to the Kennel Club database via its unique Kennel Club number to verify the information provided in the survey and to extract additional demographic data including the date of birth. Only dogs with a verified Kennel Club registration were included in the analyses. Data on individual dogs were anonymised prior to analysis. Age at death was calculated as the difference between the recorded date of birth on the Kennel Club database and the date of death provided by the owner.

Each dog had a single primary cause of death extracted from the survey free text that was mapped to a standardised disorder list developed from the VeNom coding system [21]. For dogs recorded with multiple causes of death in the free-text and where no primary cause was identifiable, the first stated cause of death was assigned. 'Old age' was assigned when either "age" or "old age" was stated as the sole cause of death. 'Old age combinations' was assigned as cause of death when "age" or "old age" was stated together with free text indicative of frailty and decline, such as arthritis, incontinence, heart failure, progressive loss of mobility / collapsing / hind legs failing, failing appetite etc. Dogs with senility, dementia or cognitive dysfunction stated as the sole cause of death were coded as 'senile dementia/cognitive dysfunction'. A category called 'unknown' was used when the stated cause of death could not be confidently determined or when the words "unknown," "undiagnosed" or "died" were used.

\section{Statistical analysis}

Data analyses used R (an online open-access language and environment for statistical computing and graphics) [22] and Matlab [23]. Proportional mortality estimates for specific causes of death were calculated by dividing the number of deaths from that cause by the total number of deaths in the same cohort. The Wilson approximation method was used to calculate 95\% confidence intervals [24]:

$$
\frac{\left(2 n p+z^{2} \pm z \sqrt{\left(z^{2}+4 n p q\right)}\right)}{2\left(n+z^{2}\right)}
$$

where $n$ is the number of reported deaths, $p$ is the reported incidence of the cause of death in question, $q$ is the deaths due to a separate cause $(1-p)$, and $z$ is the $1-\alpha / 2$ point of the standard Normal distribution (1.96 for $95 \%$ confidence interval $[95 \% \mathrm{CI}])$. 
The 'overall proportional mortality' (OPM) estimates for specific causes of death were reported based on all dogs included in the survey. The 'within breed proportional mortality' (WBPM) for the subset of causes of death with $\geq 50$ deaths ascribed in the overall study ( $n=$ 25 causes) was reported for those breeds with $\geq 50$ unique deaths reported ( $\mathrm{n}=25$ breeds). Differences between OPM and WBPM ( $\mathrm{n}=25$ breeds) were assessed for each of the 25 common causes of death using the chi-squared test with Holm adjusted $P$-values to account for multiple testing [25].

The median age at death was reported overall and for each of the 25 breeds with $\geq 50$ unique deaths, to mitigate the disproportionate effects excessively large and small values have on the mean [26]. The median age at death was also reported for each of the 25 common causes of death (with $\geq 50$ reports overall). The approximate $95 \% \mathrm{CI}$ of the median, as used to depict the 'notch' on the Box and Whisker plot, was calculated as:

$$
\frac{1.57 \times I Q R}{\sqrt{n}}
$$

where $I Q R$ is the interquartile range and $n$ is the number of responses [27].

\section{Results}

The mortality survey collected responses from 4287 owners representing 5663 deceased dogs across 179 breeds ( $82.3 \%$ of the 215 breeds recognised by the Kennel Club $^{1}$ ). The median count of deaths per breed was 11 (IQR: 4-28.5 range 1-728, full list reported in Additional file 1). Twenty-five breeds had $\geq 50$ reported deaths (Labrador Retriever, Golden Retriever, German Shepherd Dog, Cocker Spaniel, Flat Coated Retriever, English Springer Spaniel, Cavalier King Charles Spaniel, Boxer, Border Collie, Dobermann, Border Terrier, Irish Setter, West Highland White Terrier, Bernese Mountain Dog, Miniature Schnauzer, Rottweiler, Weimaraner, Staffordshire Bull Terrier, Shetland Sheepdog, Whippet, Gordon Setter, Newfoundland, Bearded Collie, Dalmatian and Pointer) and were included as individual breeds in the analyses.

\section{Longevity}

The overall median age at death across all breeds was 124 months [ 10.33 years] (95\% CI: 122.57 to 125.43 months, IQR: 86-154 months, range: 0-317 months). The distribution of age at death across all breeds is shown in Fig. 1. Of all deaths reported, 79.58\% involved euthanasia and 5.56\% had a post-mortem.

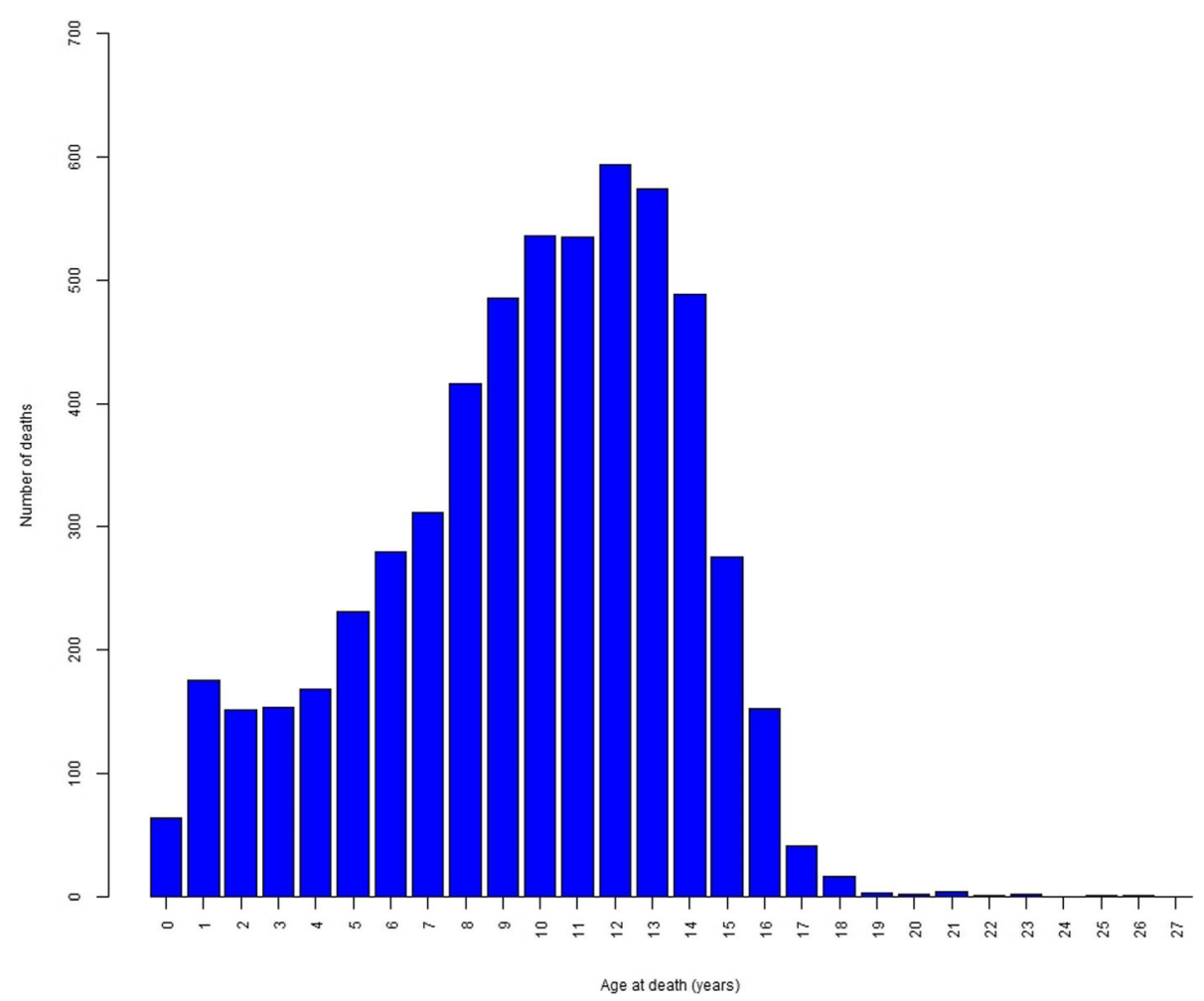

Fig. 1 Distribution of age at death from all dogs in the survey. The distribution of age at death (in years) from all dogs in the survey $(n=5663$ ) 
The median longevity varied widely across the 25 breeds with $\geq 50$ deaths reported and ranged from 152.5 months [12.71 years] in the West Highland Terrier to 92 months [7.67 years] in the Dobermann Pinscher (Table 1). The upper boundary of the approximate 95\% confidence interval of median age of death was lower than 122.57 months (the lower boundary of the approximate $95 \%$ confidence interval of the median using all data) in six of the twenty-five breeds (Flat Coated Retriever, German Shepherd Dog, Boxer, Rottweiler, Bernese Mountain Dog and Dobermann) highlighting these as shorter lived breeds. The lower boundary of the approximate $95 \%$ confidence interval of median age at death was higher than 125.43 months (the upper boundary of the approximate $95 \%$ confidence interval of the median using all data) in eight of the twenty-five breeds
(West Highland White Terrier, Bearded Collie, Border Terrier, Border Collie, Gordon Setter, Golden Retriever, Labrador Retriever, English Springer Spaniel) highlighting these as longer lived breeds. A notched box and whisker plot (Fig. 2) shows variations in the distribution of age at death within breeds, for the 25 breeds with $\geq 50$ deaths reported ('notches' in the boxes indicate the approximate $95 \%$ confidence interval of the median).

\section{Mortality}

There were 206 unique causes of death reported across the 5663 deceased dogs. The most frequently reported specific causes of death were 'old age' $(n=780, \mathrm{OPM}=$ $13.77 \%)$, 'cancer - unspecified' $(n=492, \mathrm{OPM}=8.69 \%)$ and 'heart failure' $(n=277, \mathrm{OPM}=4.89 \%)$. There were also 290 dogs recorded with 'unknown' cause of death

Table 1 Age at death statistics of the 25 breeds (with > 50 reported deaths), including the number of deaths reported (N), the contribution per breed (as a percentage of all deaths reported in the survey), the median, inter-quartile range (IQR), range (minimum and maximum) and $95 \%$ confidence interval of the median of age of death in months

\begin{tabular}{|c|c|c|c|c|c|c|}
\hline \multirow[t]{2}{*}{ Breed } & \multirow{2}{*}{$\begin{array}{l}\text { No. Deaths } \\
\text { (N) }\end{array}$} & \multirow{2}{*}{$\begin{array}{l}\% \text { of } \\
\text { all deaths }\end{array}$} & \multicolumn{4}{|c|}{ Parameters of age at death (months) by breed (with $n \geq 50$ ) } \\
\hline & & & Median & $\mathrm{IQR}$ & Range & $95 \% \mathrm{Cl}$ \\
\hline Labrador Retriever & 728 & 12.86 & 138 & $94-160$ & $2-242$ & 3.86 \\
\hline Golden Retriever & 373 & 6.59 & 141 & $102-162$ & $4-254$ & 4.91 \\
\hline German Shepherd Dog & 279 & 4.93 & 114 & $90-138$ & $4-234$ & 4.54 \\
\hline Cocker Spaniel & 266 & 4.70 & 129 & $82-155$ & $7-200$ & 7.07 \\
\hline Flat Coated Retriever & 225 & 3.97 & 114 & $90-136$ & $18-180$ & 4.85 \\
\hline English Springer Spaniel & 224 & 3.96 & 135.5 & $87.5-165$ & $3-210$ & 8.18 \\
\hline Cavalier King Charles Spaniel & 222 & 3.92 & 117 & $90-145$ & $7-255$ & 5.83 \\
\hline Boxer & 170 & 3.00 & 105.5 & $78-131$ & $6-165$ & 6.42 \\
\hline Border Collie & 119 & 2.10 & 143 & $107.5-169$ & $1-206$ & 8.91 \\
\hline Dobermann & 99 & 1.75 & 92 & $63.75-117.25$ & $7-214$ & 8.50 \\
\hline Border Terrier & 98 & 1.73 & 145 & $97-171$ & $6-228$ & 11.81 \\
\hline Irish Setter & 98 & 1.73 & 133 & $93-155$ & $6-200$ & 9.90 \\
\hline West Highland White Terrier & 96 & 1.70 & 152.5 & $110.5-175$ & $2-267$ & 10.40 \\
\hline Bernese Mountain Dog & 78 & 1.38 & 96.5 & $72-115$ & $3-178$ & 7.69 \\
\hline Miniature Schnauzer & 76 & 1.34 & 118.5 & $72.5-159$ & $4-202$ & 15.68 \\
\hline Rottweiler & 76 & 1.34 & 100.5 & $75-127.5$ & $5-173$ & 9.52 \\
\hline Weimaraner & 74 & 1.31 & 124 & $98-155$ & $0-186$ & 10.47 \\
\hline Staffordshire Bull Terrier & 71 & 1.25 & 130 & $100.25-160.5$ & $3-297$ & 11.30 \\
\hline Shetland Sheepdog & 56 & 0.99 & 135.5 & $114-162$ & $11-191$ & 10.14 \\
\hline Whippet & 55 & 0.97 & 118 & $81.5-146$ & 3-199 & 13.74 \\
\hline Gordon Setter & 54 & 0.95 & 142.5 & 119-158 & $49-178$ & 8.39 \\
\hline Newfoundland & 53 & 0.94 & 113 & $90-137$ & $2-171$ & 10.20 \\
\hline Bearded Collie & 52 & 0.92 & 149.5 & $117.5-176.5$ & $19-213$ & 12.93 \\
\hline Dalmatian & 52 & 0.92 & 130 & $80-165$ & $1-187$ & 18.62 \\
\hline Pointer & 50 & 0.88 & 134.5 & $86-152$ & 8-194 & 14.75 \\
\hline Cumulative total & 3744 & 66.11 & & - & - & \\
\hline All responses from all breeds & 5663 & $100 \%$ & 124 & $86-154$ & $0-317$ & 1.43 \\
\hline
\end{tabular}




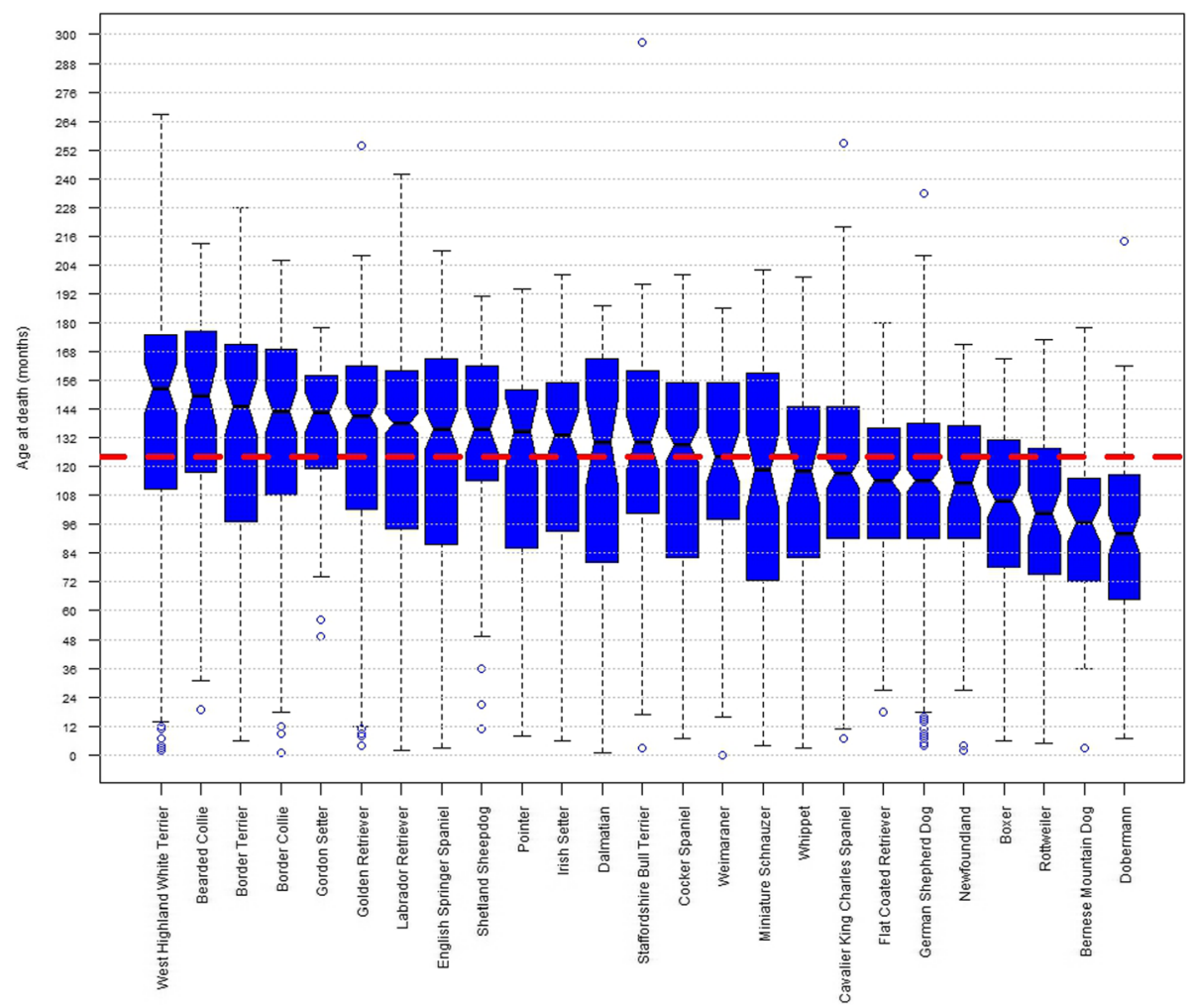

Fig. 2 Box and Whisker plot of age at death across breeds. Notched Box and Whisker plot of age at death (months) across breeds, for the 25 breeds with $\geq 50$ deaths reported. 'Notches' in the boxes indicate the approximate $95 \%$ confidence interval of the median. The red dashed line indicates the median age at death of all dogs in the survey $(n=5663)$ of 124 months, and the thickness of this line approximates to the $95 \%$ confidence interval (122.57 to 125.43 months)

$(\mathrm{OPM}=5.12 \%)$. The total number of reports and overall proportional mortality (with median, IQR and range, and $95 \%$ confidence intervals) of the 30 most common causes of death are listed in Table 2 (the full list is reported in Additional file 1).

The WBPM (and 95\% CI) for the causes of death with $\geq 50$ reports $(n=25)$ are shown for the 25 breeds with $\geq 50$ reports ( $n=3744$ deaths) (Table 3 ). The WBPM for "old age" ranged from 3.85\% in Bernese Mountain Dogs to $25.0 \%$ in Bearded Collies. The WBPM for 'cancer unspecified' ranged from $0.00 \%$ in Gordon Setters to $19.56 \%$ in Flat Coated Retrievers. The WBPM for 'heart failure' ranged from $0.00 \%$ in Whippets to $19.82 \%$ in Cavalier King Charles Spaniels.

Figure 3 illustrates higher (red), and lower (green) WBPM than OPM $(P<0.05)$ for specific causes of death among the twenty-five common breeds in the study. None of the breeds showed a significant difference between WBPM and OPM for epilepsy, gastric tumour, hepatic/liver tumour, liver failure, lung tumour, mammary tumour, oral tumour, stroke and unknown. Figure 3 can be used to assess predisposition within-breeds to certain causes of death. For example, Flat Coated Retrievers had a higher WBPM of death or euthanasia due to bone tumours, unspecified cancer and splenic tumours, and a lower WBPM of death or euthanasia from old age than OPM across the whole survey. Conversely, Labrador Retrievers had a higher WBPM of death or euthanasia due to arthritis, old age and old age combinations, and a lower WBPM of death from kidney failure than OPM across the whole survey.

\section{Longevity by mortality}

The median age at death varied widely across the 25 common causes of death. Figure 4 uses a notched box and whisker plot to show variation in distribution of age at death across causes of death with $\geq 50$ deaths reported; the 'notches' in the boxes indicate the approximate $95 \%$ confidence interval of the median. The cause of death with the oldest median age at death was old age (164.5 months; 13.71 years), and the cause of death with the youngest median age at death was road traffic accident (38.5 months; 3.21 years). The upper boundary of the approximate $95 \%$ confidence interval of median age at death was lower than 122.57 months (the lower boundary of the approximate $95 \%$ confidence interval of the median using all data) for nine of the twenty-five causes (cardiomyopathy, unknown, bone tumour, brain 
Table 2 Age at death statistics from the 30 most commonly reported causes, including the number of deaths reported ( $N$ ), the contribution per cause (as a percentage of all deaths reported in the survey), the median, inter-quartile range (IQR), range (minimum and maximum) and 95\% confidence interval of the median of age of death in months

\begin{tabular}{|c|c|c|c|c|c|c|}
\hline \multirow[t]{2}{*}{ Disease } & \multirow{2}{*}{$\begin{array}{l}\text { No. Deaths } \\
\text { (N) }\end{array}$} & \multirow{2}{*}{$\begin{array}{l}\text { Prevalence } \\
\text { (\%) }\end{array}$} & \multicolumn{4}{|c|}{ Parameters of age at death (months) by breed (with $n \geq 50$ ) } \\
\hline & & & Median & IQR & Range & $95 \% \mathrm{Cl}$ \\
\hline Old Age & 780 & 13.77 & 164.5 & $148-177$ & $69-267$ & 1.64 \\
\hline Cancer - unspecified & 492 & 8.69 & 123 & $98.5-145$ & $2-255$ & 3.31 \\
\hline Unknown & 290 & 5.12 & 112 & $71-147$ & $0-241$ & 7.05 \\
\hline Heart Failure & 277 & 4.89 & 119 & $90.75-147$ & $0-207$ & 5.34 \\
\hline Kidney Failure & 239 & 4.22 & 125 & $85-152$ & $3-210$ & 6.85 \\
\hline Old Age combinations & 229 & 4.04 & 155 & 139-169 & $86-216$ & 3.13 \\
\hline Bone tumour & 192 & 3.39 & 110.5 & $85-132.5$ & $18-242$ & 5.42 \\
\hline Lymphoma & 180 & 3.18 & 95.5 & $61-126$ & $3-171$ & 7.65 \\
\hline Cardiomyopathy & 166 & 2.93 & 114 & $82-142$ & $1-220$ & 7.36 \\
\hline Brain tumour & 159 & 2.81 & 103 & $73.5-136$ & 0-195 & 7.83 \\
\hline Stroke & 136 & 2.40 & 152.5 & $129-168$ & $7-191$ & 5.28 \\
\hline Splenic tumour & 129 & 2.28 & 126 & $101.75-146.75$ & $16-180$ & 6.26 \\
\hline Hepatic liver tumour & 115 & 2.03 & 119 & $91-142$ & 20-183 & 7.51 \\
\hline Road Traffic Accident & 102 & 1.80 & 38.5 & $14-74$ & $1-176$ & 9.39 \\
\hline Liver Failure & 89 & 1.57 & 136 & $96-152.25$ & 4-195.36 & 9.42 \\
\hline Epilepsy & 88 & 1.55 & 68 & 39-96 & $4-180$ & 9.60 \\
\hline Gastric dilation-volvulus (Bloat) & 87 & 1.54 & 101 & $60-127.75$ & $14-166.68$ & 11.48 \\
\hline Aggression & 85 & 1.50 & 39 & $21-75.25$ & $7-157$ & 9.30 \\
\hline Seizure & 82 & 1.45 & 130.5 & $81-153$ & $4-192$ & 12.56 \\
\hline Gastric tumour & 78 & 1.38 & 120.5 & $95-143$ & $25-198$ & 8.59 \\
\hline Arthritis & 77 & 1.36 & 151 & $129.42-168$ & $18-219$ & 6.95 \\
\hline Oral tumour & 72 & 1.27 & 133.5 & $103.5-154$ & 27-203 & 9.40 \\
\hline Lung tumour & 55 & 0.97 & 117 & $96-135.5$ & $32-317$ & 8.42 \\
\hline Mammary tumour & 53 & 0.94 & 126 & $111-148.25$ & 78-190 & 8.08 \\
\hline Hyperadrenocorticism (Cushing's Disease) & 50 & 0.88 & 138 & $117-158$ & 69-188 & 9.16 \\
\hline Intestinal tumour & 49 & 0.87 & 127 & $93-149$ & 28-195 & 12.64 \\
\hline Skin tumour & 45 & 0.79 & 112 & $76.75-136$ & 14-179 & 13.96 \\
\hline Surgical complication & 44 & 0.78 & 68 & $31.5-107.5$ & $2-193$ & 18.10 \\
\hline Pancreatitis & 42 & 0.74 & 109 & $67-132$ & 13-191 & 15.85 \\
\hline Leukaemia & 40 & 0.71 & 94 & $60.5-114$ & $13-177$ & 13.37 \\
\hline Cumulative total & 4522 & 79.85 & & - & - & \\
\hline All responses from all breeds & 5663 & $100 \%$ & 124 & $86-154$ & $0-317$ & 1.43 \\
\hline
\end{tabular}

tumour, gastric dilation-volvulus [GDV or bloat], lymphoma, epilepsy, aggression and road traffic accident) identifying these disorders as tending to result in death at an earlier age. The lower boundary of the approximate 95\% confidence interval of median age at death was higher than 125.43 months (the upper boundary of the approximate $95 \%$ confidence interval of the median using all data) for six of the twenty-five causes (old age, old age combinations, stroke, arthritis, hyperadrenocorticism/
Cushing's disease and liver failure) identifying these disorders as tending to result in deaths at an older age.

\section{Discussion}

This study of over five thousand deaths in pedigree dogs provides up-to-date information on longevity and causes of mortality in UK Kennel Club registered dogs that can support evidence-based efforts to improve health and welfare in dogs [11]. By reporting causes of death that 


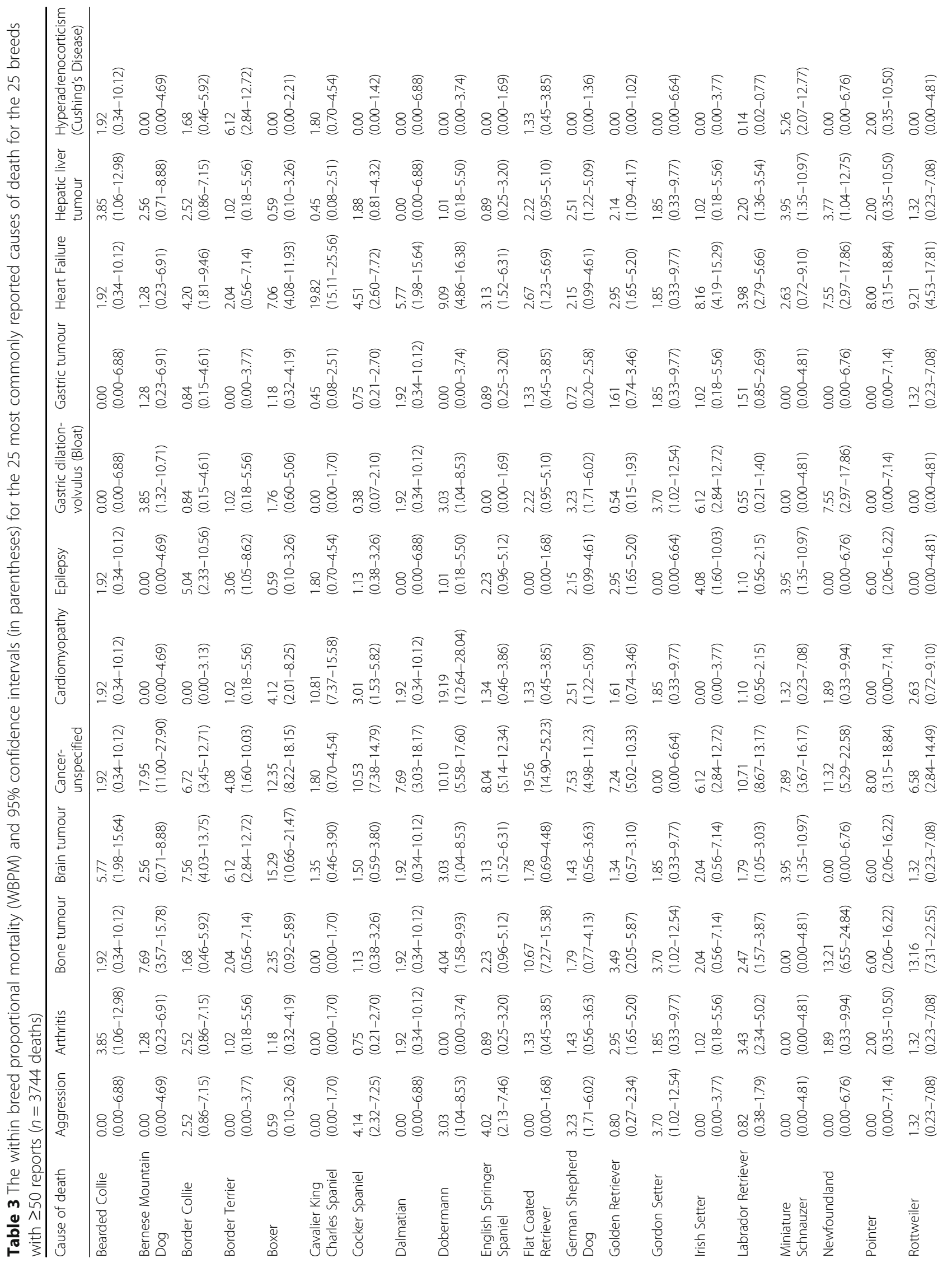




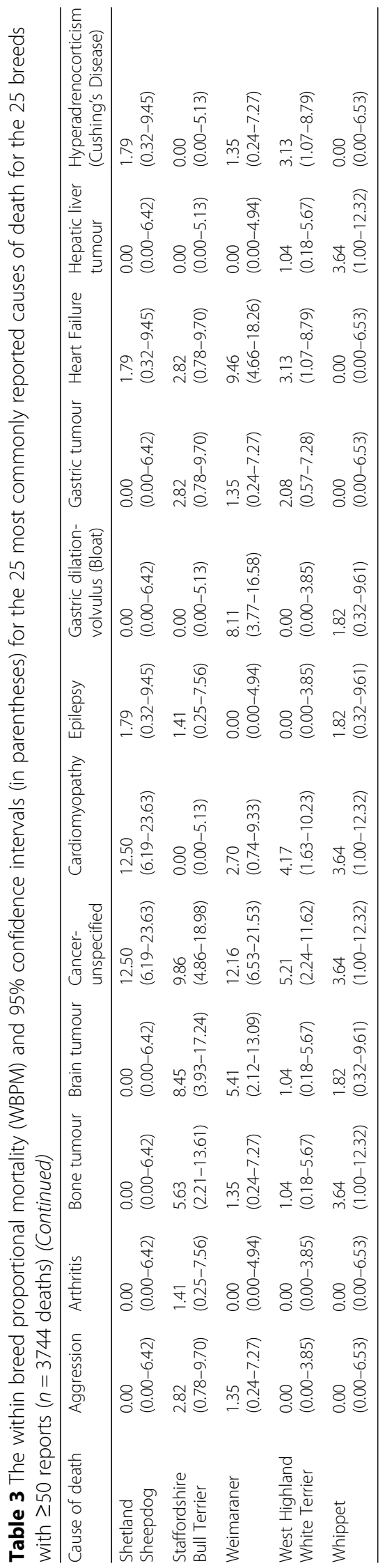




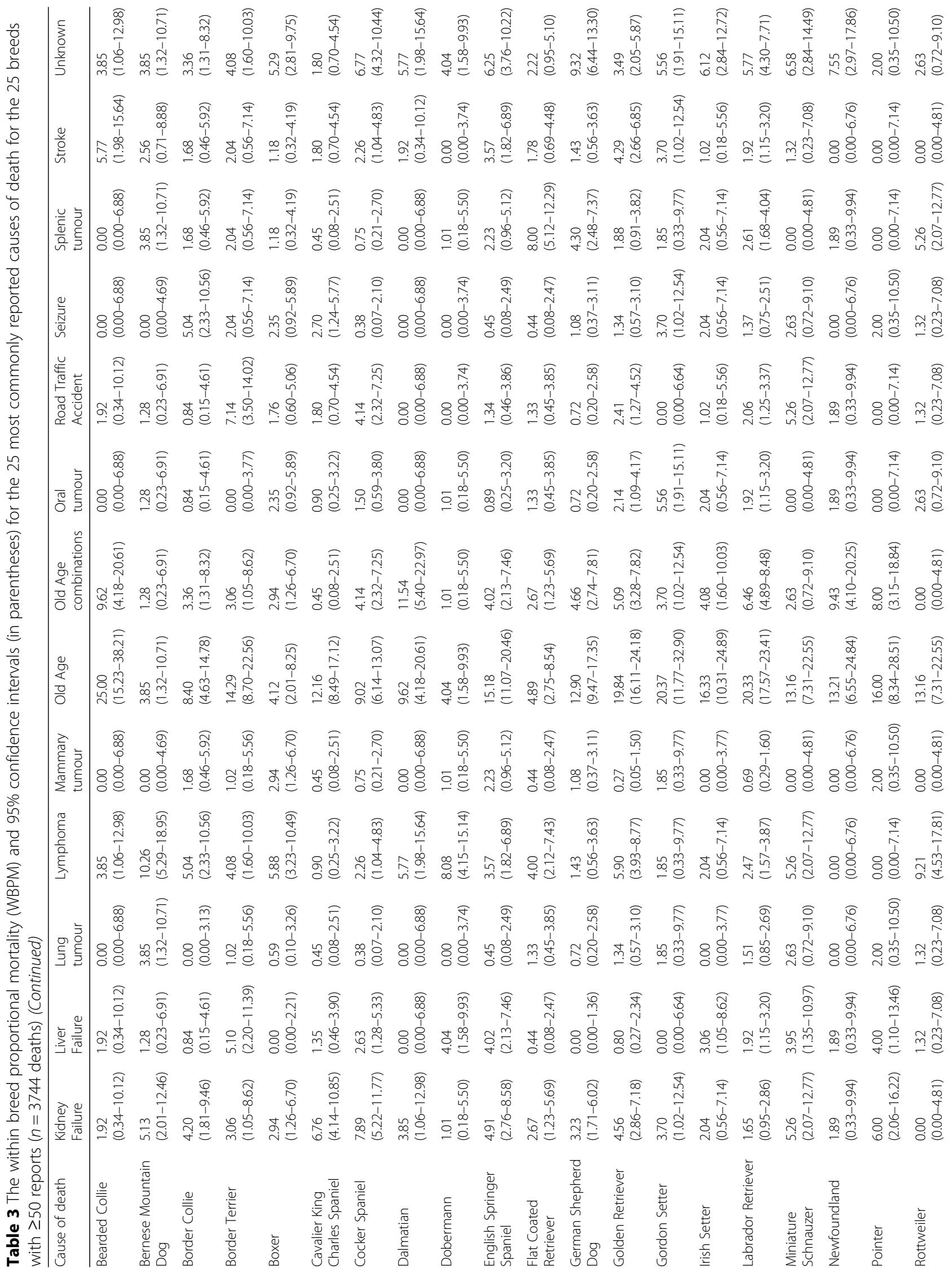




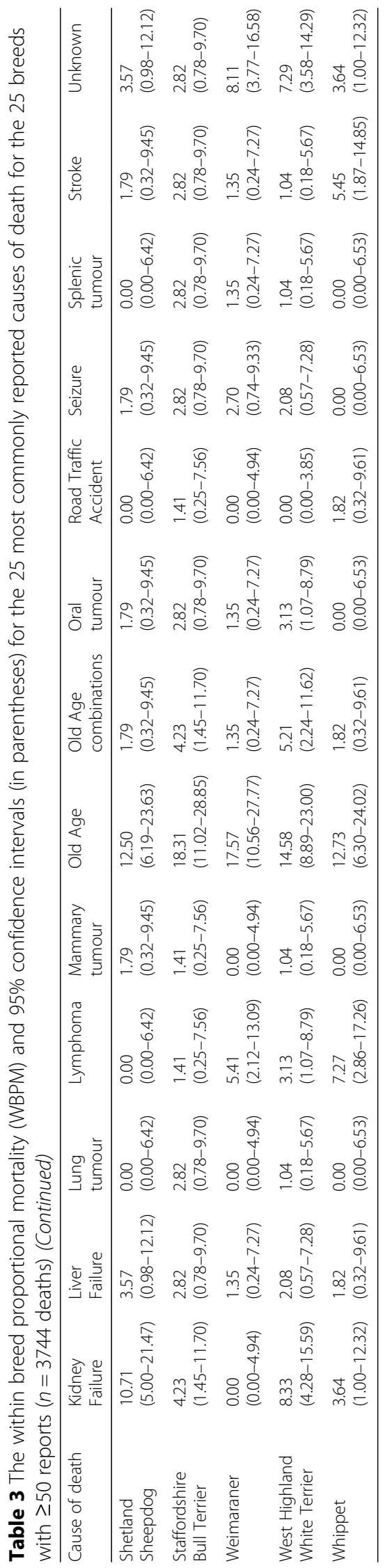




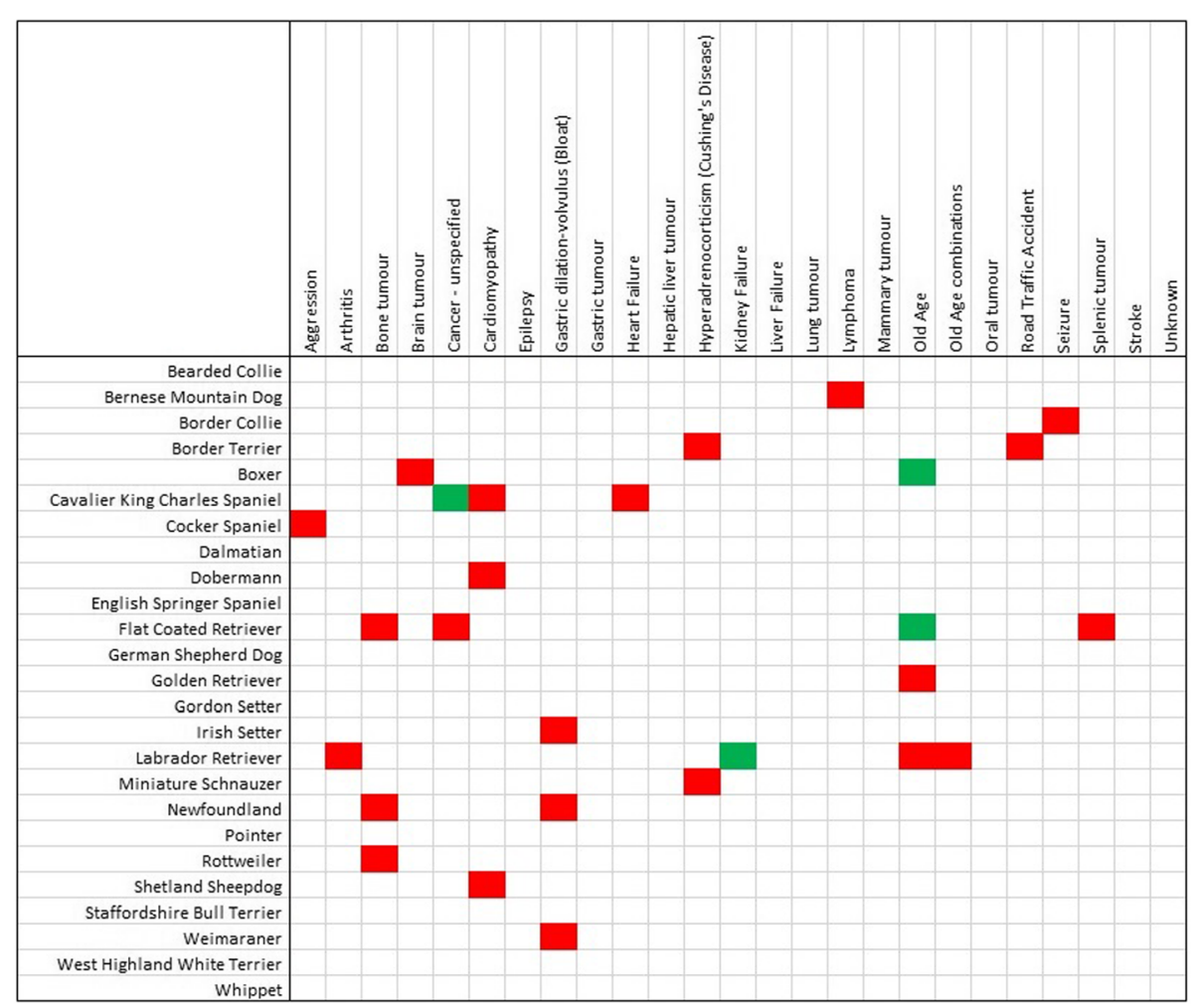

Fig. 3 Significant differences in proportional mortality within breed compared to overall. Significantly higher (red), and lower (green) within breed proportional mortality (WBPM) than overall proportional mortality $(\mathrm{OPM})(P<0.05)$ for the 25 most commonly reported causes of death among the twenty-five breeds with $>50$ deaths reported with $\geq 50$ reports ( $n=3744$ deaths)

have significantly higher and lower proportional mortality within breeds compared to all dogs, these results can support ongoing efforts to focus health reforms on priority disorders in individual breeds [28]. The results on median longevity by breed provide data that can assist current and prospective owners to manage their lifespan expectations for their dogs.

The overwhelming majority of reported deaths in dogs $(79.58 \%)$ in this study involved euthanasia. However, post-mortem examinations to determine definitively the precise condition, disease or failing that led to the death were not commonly undertaken (5.56\%). These findings concur with results reported from primary-care veterinary practice in the UK which reported that $86.4 \%$ of deaths involved euthanasia [7]. Although euthanasia entails an artificial foreshortening of lifespan, its time of occurrence often represents the approximate point at which the dog's life is adjudged by the owner and the veterinarian to no longer be 'worth' living, either for ethical reasons pertaining to impairment of quality of the dog's life and/or due to convenience in terms of management (of illness or behaviour) [29,30]. The balancing of the objectives of length and quality of life has particular poignancy for euthanasia due to behavioural rather than physical health problems (notably aggression, which accounted for 85 , or $1.5 \%$ of, deaths in the current study), since these undesirable behaviours may be substantially influenced by environmental influences unique to the individual, including failures in management and training or unrealistic expectations by owners on how their dog should behave [31, 32].

Death is an inevitable end to every life and therefore the emotional consequences for owners from the death of a companion animal are ultimately unavoidable and should be anticipated and prepared for [33]. Although death may be postponed at a population or individual level by improved healthcare, extended longevity by itself does not necessarily imply an improved or even a good quality of life, so a delicate balancing act exists between longevity and acceptable quality of life in order to optimise welfare in species where euthanasia is an option [34]. A shorter but largely healthy lifespan (the healthy component is sometimes called the healthspan [35]) with a rapid decline to death may offer better welfare than a longer but more ailed life with a slow and painful decline to death. This concept has been encompassed in the 'longevity dividend' in humans [36]. The ethical issues surrounding healthspan in dogs are exemplified by the emotional conflicts generated in owners and veterinarians during decision-making between euthanasia 


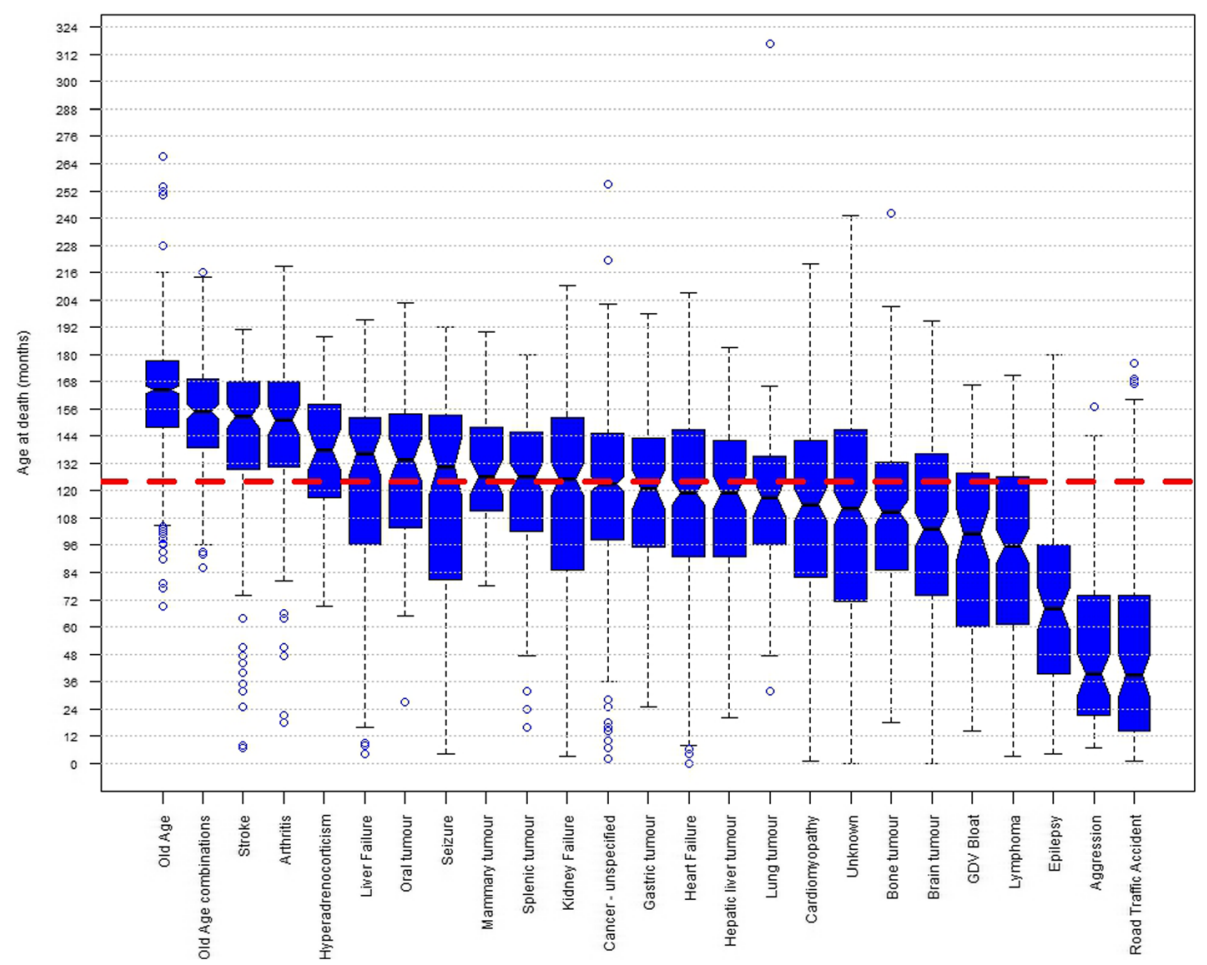

Fig. 4 Box and Whisker plot of age at death across common causes of death. Notched Box and Whisker plot of age at death (months) for the 25 causes of death with $\geq 50$ deaths reported. 'Notches' in the boxes indicate the approximate $95 \%$ confidence interval of the median. The red dashed line indicates the median age at death of all dogs in the survey $(n=5663)$ of 124 months, and the thickness of this line approximates to the $95 \%$ confidence interval (122.57 to 125.43 months)

versus waiting for unassisted death in dogs $[37,38]$. Ensuring adequate 'quality of life' of the animal is often the overriding priority for the owner and veterinarian, and once it is judged that the injury, disorder or general process of decline affecting the animal has taken sufficient toll on welfare, a decision may be made to foreshorten life for humane reasons [39]. Thus, a paradox may occur where an increased appreciation of welfare in older dogs may lead to greater use of euthanasia and therefore result in a shortening of general lifespan. Additionally, a generally shorter lifespan of some breeds compared to others should not be viewed as necessarily a welfare problem per se, provided there is a high quality of life during the living years and that the death process is relatively benign. Conversely, welfare concerns should be raised for breeds that die commonly from lengthy, debilitating and painful disorders, particularly when these are accompanied by a general foreshortened lifespan.

The current study reports that the median age at death across all breeds was 10.33 years. This is similar to previously reported estimates: 11.25 years in UK Kennel Club registered dogs [9]; 11.9 years (IQR 8.4 to 14.0 years) from primary veterinary practice data on UK 'purebred' dogs [7]; and 10 years (IQR 6 to 12 years) reported from a survey conducted by the Danish Kennel Club [10]. However, substantial variation in median longevity across breeds was also shown in the current study (Fig. 2). The longest-lived breed was the West Highland White Terrier (median age at death of 12.71 years) and the shortest was the Dobermann Pinscher (median age at death of 7.67 years). However, as discussed above, such variation in longevity is not unexpected based on previous studies that reported similar results $[7-10,18]$ and in part reflects the extent of phenotypic variation that exists across the spectrum of domestic dog breeds. Indeed, a general inverse correlation of longevity and body size is well established among dog breeds, with 'giant' breeds in general often exhibiting notably shorter life expectancy than small or miniature breeds $[7-9,40]$. Several breeds in our study had median ages at death significantly lower than the overall median longevity (Flat Coated Retriever, German Shepherd Dog, Boxer, Rottweiler, Bernese Mountain Dog and Dobermann) which may raise health concerns in these breeds and prompt exploration on common causes of death in efforts to redress longevity deficits. However, as argued above, shorter life expectancy per se need not intrinsically imply welfare impairment, on the proviso that such shorter lives are generally healthy and that the dying 
process is not protracted or malign. Conversely, breeds determined as having a longer life expectancy should not automatically be regarded as being 'healthier' since it may be that such breeds are subject to long and distressing periods of decline to the point of euthanasia or death. Therefore, in order to gain greater insights into the nature of ageing, decline and ultimately death within breeds, it is necessary to consider the welfare costs of the common specific causes of death together with variation in longevity.

Substantial variation in median longevity across specific causes of death was also identified in the current study (Fig. 4). Some of these differences are intuitive; for example, the median ages at death from 'old age' and 'old age combinations' (13.71 years and 12.92 years respectively) were higher than the overall median age at death (10.33 years) as might be predicted. Specific causes of death with higher median ages at death may be described as 'diseases of ageing' (e.g. stroke, 12.71 years; arthritis, 12.58 years). Conversely, causes of death with lower median ages at death may be viewed as life curtailing and possibly of greater welfare impact in the sense of years of potential life lost (e.g. road traffic accident, 3.21 years; aggression, 3.25 years; epilepsy, 5.67 years) [32]. These disorder-based longevity data offer insights that can assist with disorder prioritisation for reforms within breeds that can optimise the welfare gains from the effort and resources expended.

Some significant differences were identified between the within-breed proportional mortality (WBPM) from individual disorders and the overall proportional mortality (OPM) in some breeds that can assist to identify life-limiting predispositions in these breeds (Fig. 3). At least one breed had a significantly higher or lower WBPM than OPM for 16 of the 25 common causes of death analysed. Many of these associations concur with previous reports in these breeds. For example, our study determined a higher proportional mortality from cardiac disorders in the Cavalier King Charles Spaniel (WBPM of $19.82 \%$ for heart failure and $10.81 \%$ for cardiomyopathy compared to the OPM of $4.89 \%$ and $2.93 \%$ respectively) which is concordant with previous reports that cardiac conditions were the most common cause of death in this breed $[9,41,42]$. Similarly, Flat Coated Retrievers were more likely to die from bone cancer, unspecified cancer and splenic tumours, in line with previous findings of high cancer-related morbidity and mortality in this breed $[4,43-45]$. When taken with the apparently foreshortened median lifespan of Flat Coated Retrievers (9.5 years), these results imply that the breed is predisposed to these types of cancers and that this cancer predisposition likely contributes to a substantially curtailed life expectancy, which therefore may be viewed as a welfare problem. Figure 3 also revealed or confirmed other breeds at higher risk of mortality for specific conditions; for example, Irish Setter, Newfoundland and Weimaraner from GDV [46, 47], Dobermann and Cavalier King Charles Spaniel from cardiomyopathy [48, 49], Newfoundland and Rottweiler from bone cancer (in addition to the Flat Coated Retriever) [50], and Cocker Spaniel from aggression [32]. There were also some unexpected and perhaps less well established findings, such as significantly higher mortality from road traffic accidents in the Border Terrier. However, the relatively small sample sizes for some breeds and disorders mean that novel findings should be treated as hypothesis generators that are validated in later confirmatory studies [51].

Merging the results of longevity by breed with WBPM of causes of death by breed may allow assignment of breeds to one of four categories: 1) long-lived with no specific cause of death at a raised proportional mortality (e.g. West Highland White Terrier, Bearded Collie, Gordon Setter); 2) long-lived with at least one cause of death at higher proportional mortality (Labrador [old age, old age combinations and arthritis], Golden Retrievers [old age], Border Collie [seizure]); 3)

short-lived with no cause of death at a raised proportional mortality (German Shepherd Dog, Whippet); and 4) short-lived with at least one cause of death at a higher proportional mortality (Dobermann [cardiomyopathy], Bernese Mountain Dog [lymphoma], Flat Coated Retriever, Rottweiler and Newfoundland [bone cancer], Cavalier King Charles Spaniel [cardiomyopathy and heart failure]). This is shown visually in Fig. 5. Category 4 (short-lived with increased probability of at least one cause of death) could be considered to represent breed predisposition for serious life-limiting conditions and to represent potential welfare concerns, particularly where the cause of death at a raised proportional mortality has a low median age (e.g. lymphoma [7.96 years]; bone tumour [9.21 years]). Conversely categories 1 (long lived with no raised proportional mortality of a cause of death) and 3 (short lived with no raised proportional mortality of a cause of death) may represent a general variation in longevity associated with factors that apply across all dogs, such as body size, but with no obvious disease or disorder as an accentuating driver for death $[7,9]$.

The causes of death reported in this study with the highest proportional mortality across all breeds were old age (13.77\%), unspecified cancer (8.69\%) and heart failure $(4.89 \%)$. The proportional mortality due to old age was lower than that reported in a survey conducted by the Danish Kennel Club (20.8\%) [10] and an earlier UK study comprising UK Kennel Club registered dogs (17.8\%) [9]. The Danish study also reported a higher frequency cause of death due to cancer $(14.5 \%)$ than the current study [10]. Differing reported proportional mortality values between studies may reflect differences in 
disease classification; for example, cancer was often listed as the specific variant in the current study rather than as a general 'cancer' category [6]. Additionally, the current study only classified 'old age' as the cause of mortality when 'age' or 'old age' was solely cited by the respondent; when multiple disorders/conditions were listed along with 'old age' this was recorded as 'old age combinations'; adding the proportional mortality from 'old age' and 'old age combinations' in our study identifies that $17.8 \%$ of dogs died from causes relating to old age. This implies that just under a fifth of dogs in this survey died from conditions that the owner considered to be intrinsically linked to ageing and decline. The rationale for including 'old age combinations' as a cause of death was to remove the subset of the specific causes of death from the overall analyses that were considered by the owner to reflect terminal decline rather than an intrinsic pathology. For example, 'incontinence,' arthritis' and 'weak hind legs / collapse' as causes of death imply different aetiology in 'old' dogs (where they are consistent with age related decline) than in dogs in their prime of life (when they would be indicative of disease or trauma).
This study had some limitations. The nested mortality survey had low uptake compared with the originating morbidity survey; only 5663 deaths were reported compared with responses relating to 43,005 live dogs in the morbidity survey [4]. An earlier survey on pedigree dogs in the UK was more successful and gathered data on 15,881 deaths [9]. The reasons behind the current low response rate are unknown but may have introduced some response rate bias [52]. Changes to breed popularity over time are likely to also introduce some temporal biases to the results $[53,54]$. Breeds that are increasing in popularity are likely to have a proportionally greater number of deaths of younger dogs and therefore to shift the median longevity downwards while breeds that are decreasing in popularity will conversely show an apparent extension in longevity compared with the true situation because there are relatively more of the older dogs available to die $[55,56]$. The popularity of breeds registered with the Kennel Club over the last decade is known to be non-static so this may have biased the longevity estimates downwards for breeds with increasing registrations (e.g. Whippet, Cocker Spaniel and

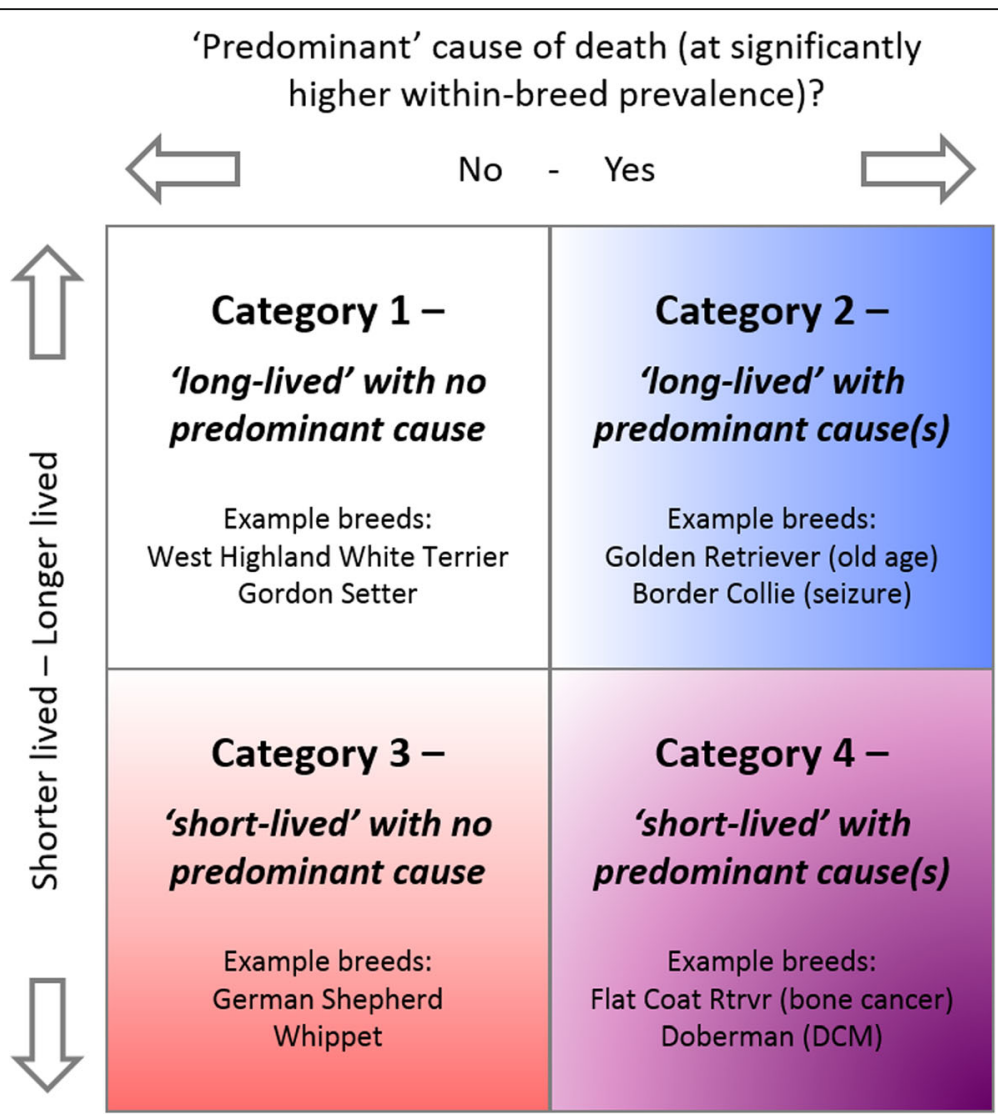

Fig. 5 Diagrammatic representation of the 4 categories breeds may be assigned to based on longevity and high within breed prevalence of particular cause(s) of death. Longer and shorter lived (red) shown on vertical; binary category on horizontal indicating no cause of death at higher within breed prevalence (left) and one or more causes at higher within breed prevalence (right) 
Miniature Schnauzer) and biased the longevity estimates upwards for breeds with decreasing registrations (e.g. West Highland White Terrier, Cavalier King Charles Spaniel and Irish Setter) [57]. However, the representation of the 25 breeds with 50 or more deaths reported was broadly in line with registered population size; the correlation of number of deaths reported and total registrations of dogs born between 2000 and 2004 (the distribution of year of birth was approximately normal with averages at 2000/2001, not shown) was 0.9. Further temporal biases or influences on longevity include technological advances and improvements in veterinary treatment and care, selection occurring against disease across the time frame that coincides with the lifespan of dogs in this study, and possibly other changes in the genetics of breed populations. The biological causes for seemingly arbitrary variation in life expectancy (i.e. where there is no obvious causal disorder) across breeds (or other categories, such as sex) are unknown and likely to be hugely complex. Exploring the full depth of these complexities was outside the aims of the current study but data gathered here and from similar studies may help to reveal new insights into dog life expectancy in future analyses.

\section{Conclusion}

Substantial variation in the median lifespan and the prominent causes of death exists across breeds. This study has identified individual breeds that have both a low median lifespan and also a high proportional mortality for one or more specific causes of death. Breeds with this combination are highlighted with potential welfare concerns that may need to be addressed.

\section{Endnote}

${ }^{1}$ Refers to the breeds recognised at the time of the survey. Six breeds have been recognised by The Kennel Club since the survey closed.

\section{Additional file}

Additional file 1: Number of reported deaths (and percentage of total reported) per breed. Number of reported deaths (and percentage [prevalence, and 95\% confidence interval]) per cause. (XLSX 24 kb)

\section{Abbreviations}

95\% Cl: 95\% confidence interval; GDV: Gastric dilation volvulus; IQR: Interquartile range; OPM: Overall proportional mortality; WBPM: Within breed proportional mortality

\section{Acknowledgements}

The authors are grateful to the many staff employed at the Kennel Club who contributed towards the conception of the study, and the compilation and distribution of the survey, including Nick Sutton, Charlotte McNamara, Bonnie-Marie Abhayaratne and Dina Ahmad. We are also grateful to Dr. Lucy Asher and Dr. Naomi Harvey from the University of Nottingham School of Veterinary Medicine and Science for contributing towards the questions in their respective fields. The authors also gratefully acknowledge the time taken by all participants in the survey.

Funding

This study was funded internally by the Kennel Club.

\section{Availability of data and materials}

The datasets generated during and/or analysed during the current study are available from the corresponding author on reasonable request.

\section{Authors' contributions}

$A L-Z$ and BW first conceived the idea for the study. BW compiled the survey with help from AL-Z, TL, DON and KE and conducted preliminary data processing. TL performed the statistical analysis. The paper was written primarily by $T L, B W$ and DON with additional contributions from AL-Z and KE. All authors have approved the final article.

\section{Ethics approval and consent to participate}

Participants consented to the data provided on dogs being used for the purposes of research according to the Kennel Club's Privacy policy: http://www.thekennelclub.org.uk/privacy-policy/?utm_campaign=PBHS +EMAIL++Single+dog+owners\&utm_source=emailCampaign\&utm_medium =email\&utm_content=

\section{Consent for publication}

Not applicable.

\section{Competing interests}

$\mathrm{KE}$ and $\mathrm{TL}$ are employed by the Kennel Club. BW and AL-Z were employed by the Kennel Club and KE was employed by the University of Nottingham School of Veterinary Medicine and Science at the time the study was undertaken. DON was funded at the RVC by an award from the Kennel Club Charitable Trust.

\section{Publisher's Note}

Springer Nature remains neutral with regard to jurisdictional claims in published maps and institutional affiliations.

\section{Author details}

${ }^{1}$ The Kennel Club, Clarges Street, London W1J 8AB, UK. ${ }^{2}$ School of Veterinary Medicine and Science, The University of Nottingham, Sutton Bonington Campus, Sutton Bonington, Loughborough LE12 5RD, UK. ${ }^{3}$ International Partnership for Dogs, 504547 Grey Rd 1, Georgia Bluffs, ON, Canada. ${ }^{4}$ Pathobiology and Population Health, The Royal Veterinary College, Hawkshead Lane, North Mymms, Hatfield AL9 7TA, UK.

Received: 30 July 2018 Accepted: 27 September 2018

Published online: 17 October 2018

\section{References}

1. Wayne RK, Leonard JA, Vila C. Genetic analysis of dog domestication. In: Zeder MA, editor. Documenting domestication: new genetic and archaeological paradigms. Berkeley, California: University of California Press; 2006. p. 279-93.

2. Spady TC, Ostrander EA. Canine behavioral genetics: pointing out the phenotypes and herding up the genes. Am J Hum Genet. 2008;82(1):10-8.

3. Vaysse A, Ratnakumar A, Derrien T, Axelsson E, Rosengren Pielberg G, Sigurdsson $\mathrm{S}$, et al. Identification of genomic regions associated with phenotypic variation between dog breeds using selection mapping. PLoS Genet. 2011;7(10):e1002316.

4. Wiles BM, Llewellyn-Zaidi AM, Evans KM, O'Neill DG, Lewis TW. Large-scale survey to estimate the prevalence of disorders for 192 Kennel Club registered breeds. Canine Genet Epidemiol. 2017;4(1):8.

5. Gough A, Thomas A, O'Neill D. Breed predispositions to disease in dogs and cats. 3rd ed. Chichester, West Sussex: Wiley-Blackwell; 2018. p. 398.

6. O'Neill DG, Church DB, McGreevy PD, Thomson PC, Brodbelt DC. Prevalence of disorders recorded in dogs attending primary-care veterinary practices in England. PLoS One. 2014;9(3):1-16.

7. O'Neill DG, Church DB, McGreevy PD, Thomson PC, Brodbelt DC. Longevity and mortality of owned dogs in England. Vet J. 2013;198(3):638-43. 
8. Patronek GJ, Waters DJ, Glickman LT. Comparative longevity of pet dogs and humans: implications for gerontology research. J Gerontol. 1997;52(3):B171-B8.

9. Adams VJ, Evans KM, Sampson J, Wood JLN. Methods and mortality results of a health survey of purebred dogs in the UK. J Small Anim Pract. 2010 51(10):512-24.

10. Proschowsky HF, Rugbjerg H, Ersbøll AK. Mortality of purebred and mixedbreed dogs in Denmark. Prev Vet Med. 2003;58(1-2):63-74

11. Bateson P. Independent inquiry into dog breeding. Cambridge: University of Cambridge; 2010.

12. O'Neill DG, Coulson NR, Church DB, Brodbelt DC. Demography and disorders of German shepherd dogs under primary veterinary care in the UK. Canine Genet Epidemiol. 2017;4(1):7.

13. O'Neill DG, Seah WY, Church DB, Brodbelt DC. Rottweilers under primary veterinary care in the UK: demography, mortality and disorders. Canine Genet Epidemiol. 2017;4(1):13.

14. O'Neill DG, Darwent EC, Church DB, Brodbelt DC. Border terriers under primary veterinary care in England: demography and disorders. Canine Genet Epidemiol. 2017:4(1):15.

15. O'Neill D, Church D, McGreevy P, Thomson P, Brodbelt DC. Approaches to canine health surveillance. Canine Genet Epidemiol. 2014;1(1):2.

16. Bonnett BN, Egenvall A. Age patterns of disease and death in insured Swedish dogs, cats and horses. J Comp Pathol. 2010;142(Supplement 1): S33-S8.

17. Bonnett BN, Egenvall A, Hedhammar $\AA$, Olson P. Mortality in over 350,000 insured Swedish dogs from 1995-2000: I. breed-, gender-, age- and causespecific rates. Acta Vet Scand. 2005;46(3):105-20.

18. Egenvall A, Bonnett BN, Hedhammar A, Olson P. Mortality in over 350,000 insured Swedish dogs from 1995-2000: II. breed-specific age and survival patterns and relative risk for causes of death. Acta Vet Scand. 2005;46(3): $121-36$

19. Egenvall A, Nødtvedt A, Penell J, Gunnarsson L, Bonnett BN. Insurance data for research in companion animals: benefits and limitations. Acta Vet Scand. 2009;51:42.

20. Egenvall A, Bonnett BN, Olson P, Hedhammar Å. Validation of computerized Swedish dog and cat insurance data against veterinary practice records. Prev Vet Med. 1998;36(1):51-65.

21. The VeNom Coding Group. VeNom Veterinary Nomenclature [http://www.venomcoding.org].

22. R: A language and environment for statistical computing [Internet]. 2018 [cited July 1st, 2018]. Available from: http://www.R-project.org/.

23. Inc. TM. MATLAB and statistics toolbox release 2012b. Natick, Massachusetts, United States: The MathWorks Inc.; 2018.

24. Newcombe RG. Two-sided confidence intervals for the single proportion: comparison of seven methods. Stat Med. 1998;17(8):857-72.

25. Aickin $\mathrm{M}$, Gensler $\mathrm{H}$. Adjusting for multiple testing when reporting research results: the Bonferroni vs Holm methods. Am J Public Health. 1996:86:726-8.

26. Kirkwood BR, Sterne JAC. Essential medical statistics. 2nd ed. Oxford: Blackwell Science; 2003.

27. Chambers JM, Cleveland WM, Kleiner B, Tukey PA. Comparing data distributions. In graphical methods for data analysis, 62. Belmont, California: Wadsworth International Group; 1983. isbn:087150-413-8.

28. The Kennel Club. The Kennel Club's Breed Health and Conservation Plans project. https://www.thekennelclub.org.uk/health/breed-health-andconservation-plans/.

29. Trapp SM, lacuzio Al, Barca Junior FA, Kemper B, Silva LCD, Okano W, Tanaka NM, Grecco FCDAR, Cunha Filho LFCD, Sterza FDAM. Causes of death and reasons for euthanasia in a hospital population of dogs and cats. Braz J Vet Res Anim Sci. 2010;47:395-402.

30. Rollin BE. Euthanasia and quality of life. J Am Vet Med Assoc. 2006;228: 1014-6.

31. Jagoe A, Serpell J. Owner characteristics and interactions and the prevalence of canine behaviour problems. Appl Anim Behav Sci. 1996:47(1/2):31-42

32. Boyd C, Jarvis S, McGreevy PD, Heath S, Church DB, Brodbelt DC, O'Neill DG Mortality resulting from undesirable behaviours in dogs aged under three years attending primary-care veterinary practices in England. Anim Welf. 2018:27:251-62

33. Davis H, Irwin P, Richardson M, O'Brien-Malone A. When a pet dies: religious issues, euthanasia and strategies for coping with bereavement. Anthrozoos. 2003;16(1):57-74.
34. Reynolds CA, Oyama MA, Rush JE, Rozanski EA, Singletary GE, Brown DC et al. Perceptions of quality of life and priorities of owners of cats with heart disease. J Vet Intern Med. 2010;24(6):1421-6.

35. Martin-Montalvo A, Mercken EM, Mitchell SJ, Palacios HH, Mote PL, Scheibye-Knudsen $\mathrm{M}$, et al. Metformin improves healthspan and lifespan in mice. Nat Commun. 2013;4:2192

36. Creevy KE, Austad SN, Hoffman JM, O'Neill DG, Promislow DEL. The companion dog as a model for the longevity dividend. Cold Spring Harb Perspect Med. 2016;6(1):a026633.

37. Yeates JW, Main DCJ. Veterinary opinions on refusing euthanasia: justifications and philosophical frameworks. Vet Rec. 2011;168(10):263.

38. Yeates J. Ethical aspects of euthanasia of owned animals. Practice. 2010; 32(2):70-3.

39. Tzivian L, Friger M, Kushnir T. Associations between stress and quality of life: differences between owners keeping a living dog or losing a dog by euthanasia. PLoS One. 2015;10(3):e0121081.

40. Galis F, Van Der Sluijs I, Van Dooren TJM, Metz JAJ, Nussbaumer M. Do large dogs die young? J Exp Zool B Mol Dev Evol. 2007;308B(2):119-26.

41. Mattin MJ, Boswood A, Church DB, López-Alvarez J, McGreevy PD, O'Neill $\mathrm{DG}$, et al. Prevalence of and risk factors for degenerative mitral valve disease in dogs attending primary-care veterinary practices in England. J Vet Intern Med. 2015;29(3):847-54.

42. Summers J, O'Neill D, Church D, Thomson P, McGreevy P, Brodbelt D. Prevalence of disorders recorded in cavalier King Charles spaniels attending primary-care veterinary practices in England. Canine Genet Epidemiol. 2015;2(1):4.

43. Constantino-Casas F, Mayhew D, Hoather TM, Dobson JM. The clinical presentation and Histopathologic-Immunohistochemical classification of Histiocytic sarcomas in the flat coated retriever. Vet Pathol Online. 2011; 48(3):764-71.

44. Dobson J, Hoather T, McKinley TJ, Wood JL. Mortality in a cohort of flatcoated retrievers in the UK. Vet Comp Oncol. 2009;7(2):115-21.

45. Egenvall A, Nodtvedt A, von Euler H. Bone tumors in a population of 400 000 insured Swedish dogs up to $10 \mathrm{y}$ of age: incidence and survival. Can J Vet Res. 2007;71(4):292-9.

46. O'Neill DG, Case J, Boag AK, Church DB, McGreevy PD, Thomson PC, Brodbelt DC. Gastric dilation-volvulus in dogs attending UK emergency-care veterinary practices: prevalence, risk factors and survival. J Small Anim Pract. 2017;58:629-38.

47. Glickman LT. Incidence of and breed-related risk factors for gastric dilatation-volvulus in dogs. J Am Vet Med Assoc. 2000;216:40-5.

48. Martin MWS, Stafford Johnson MJ, Celona B. Canine dilated cardiomyopathy: a retrospective study of signalment, presentation and clinical findings in 369 cases. J Small Anim Pract. 2009:50:23-9.

49. Mattin MJ, Boswood A, Church DB, López-Alvarez J, McGreevy PD, O'Neill DG, Thomson PC, Brodbelt DC. Prevalence of and risk factors for degenerative mitral valve disease in dogs attending primary-care veterinary practices in England. J Vet Intern Med. 2015;29:847-54.

50. Rosenberger JA, Pablo NV, Crawford PC. Prevalence of and intrinsic risk factors for appendicular osteosarcoma in dogs: 179 cases (1996-2005). J Am Vet Med Assoc. 2007;231:1076-80.

51. Dohoo I, Martin W, Stryhn H. Veterinary epidemiologic research. 2nd ed. Charlottetown: VER Inc; 2009

52. Scott A, Jeon S-H, Joyce CM, Humphreys JS, Kalb G, Witt J, et al. A randomised trial and economic evaluation of the effect of response mode on response rate, response bias, and item non-response in a survey of doctors. BMC Med Res Methodol. 2011;11(1):126.

53. Vrac M, Friederichs P. Multivariate - Intervariable, spatial, and temporal Bias correction. J Clim. 2014:28(1):218-37.

54. Boakes EH, McGowan PJK, Fuller RA, Chang-qing D, Clark NE, O'Connor K, et al. Distorted views of biodiversity: spatial and temporal Bias in species occurrence data. PLoS Biol. 2010;8(6):e1000385.

55. Urfer SR. Bias in canine lifespan estimates through right censored data. J Small Anim Pract. 2011:52(10):555.

56. Urfer SR. Right censored data ('cohort bias') in veterinary life span studies. Vet Rec. 2008;163(15):457-8.

57. The Kennel Club. Breed registration statistics [http://www.thekennelclub.org. uk/registration/breed-registration-statistics/]. 